\title{
Liner radius fluctuations in a high-gain Cherenkov free-electron laser
}

\author{
I. de la Fuente, ${ }^{*}$ P. J. M. van der Slot, and K.-J. Boller \\ Laser Physics and Non-Linear Optics Group, Department of Science and Technology, University of Twente, P.O. Box 217, \\ 7500 AE Enschede, The Netherlands
}

(Received 15 December 2006; published 2 February 2007)

\begin{abstract}
Phase shifts in the propagating electromagnetic field of a Cherenkov free-electron laser (CFEL) can affect its gain. The phase velocity of an electromagnetic wave varies, for example, when the lined waveguide is inhomogeneous along its length. In this paper, we study quantitatively the saturated power of a particular CFEL at both weak and strong electron-beam pumping when the inner radius of the liner contains fluctuations along the waveguide. We show that the gain bandwidth of the CFEL is substantially broadened when the CFEL is pumped with a high-current beam. We also show that the design of a CFEL needs to include optimization with respect to sensitivity to liner fluctuations, especially for weakly pumped CFELs, that is, CFELs that use a low-current electron-beam density. This optimization can be relaxed for more strongly pumped CFELs.
\end{abstract}

DOI: 10.1103/PhysRevSTAB.10.020702

PACS numbers: 41.60.Cr

\section{INTRODUCTION}

The simplicity, high efficiency, and compact size of Cherenkov free-electron lasers (CFELs) make them attractive candidates for applications where widely tunable highfrequency microwaves with high powers are needed $[1,2]$. CFELs have been operated at $100 \mathrm{~kW}$ peak power level at $1 \mathrm{~mm}$ wavelengths [3], at $200 \mathrm{MW}$ peak power level at $8 \mathrm{~cm}$ wavelengths [4], and radiation has been produced at wavelengths as short as in the far infrared [5]. A typical CFEL comprises an electron beam copropagating with an electromagnetic wave through a dielectric loaded waveguide [6]. The dielectric loaded waveguide, also called lined waveguide, reduces the phase velocity of the wave to match it to the electron-beam velocity. Furthermore, modes with a longitudinal component of the electric field can exchange energy with the electrons. Upon further propagation, the energy modulation of the electron beam becomes a charge density modulation and the electrons form bunches at the scale of the radiation wavelength. For a certain relative phase between these bunches and the electromagnetic wave, the bunches are decelerated thereby amplifying the field amplitude. The contributions to the field amplitude from the different bunches add up in phase and the field is amplified exponentially until saturation sets in after a certain interaction length, i.e., when the bunch shifts out of the phase range corresponding to gain into the phase range corresponding to absorption of the field.

The gain of a CFEL, and any other coherent electronbeam-based radiation source, is determined by how well the bunches are formed within the electron beam and how well the phase matching of these bunches with the radiation field is maintained during propagation. Therefore, the gain is degraded by an energy spread in the electron beam as the varying electron velocity reduces the bunching of the

*Electronic address: i.delafuentevalentin@utwente.nl electrons [7]. Similarly, it can be expected that, if the phase velocity of the radiation wave fluctuates, then both the formation of the bunches and the phase matching are adversely affected. Such phase velocity fluctuations can be present in CFELs with nonuniform lined waveguides, e.g., when the dielectric constant varies or when the liner or waveguide radii are nonconstant along the length of the device. For the specific case of low-current pumped CFELs, we have shown that fluctuations in the wave phase velocity should be minimized in order to avoid significant degradation of the gain of the device [8]. In contrast to this, when pumping with a high beam current, it might be expected that the resulting high gain would allow the CFEL to tolerate liner irregularities. However, the realization of a high electron-beam current can technically be much more difficult than minimizing liner irregularities because space-charge effects grow strongly with the current density.

In this paper, we investigate the single pass saturated power of CFELs also in the high-gain regime when liner irregularities are present. We find that high-current pumping of the CFEL produces a broadband gain bandwidth extending over frequencies that, for a reduced current, are far from resonance. The effect of the fluctuations in the liner radius are a reduction of the maximum attainable saturation power and a broadening of the gain curve in both gain regimes even for small fluctuations.

\section{CFEL DYNAMICS}

The model that describes the basic dynamics of the electromagnetic field and the electron beam in a CFEL in the presence of liner radius fluctuations has been presented in our previous work [8], therefore we only summarize the results here. To describe the guided wave propagation, we expand the electric field as a superposition of $\mathrm{TM}_{0 n}$ modes in the cold lined waveguide (waveguide in the absence of 
the electron beam). However, to include any amplitude growth, or damping, and phase shift of the radiation through interaction with the electron beam, we allow for a spatial dependence of the amplitude $a_{n}(z)$ and phase $\phi_{n}(z)$ of the modes. For a quasimonochromatic probe input field at frequency $\omega$ propagating along the axis of the lined waveguide ( $z$-axis), we can write the field in the lined waveguide as

$$
\vec{E}(\vec{r}, t)=\sum_{n=1}^{\infty} \overrightarrow{\mathcal{E}}_{n}(r) a_{n}(z) e^{i \phi_{n}(z)} e^{i\left(k_{z n} z-\omega t\right)},
$$

where $\overrightarrow{\mathcal{E}}_{n}$ are the transversal eigenmodes expressed as a combination of Bessel functions [9] with longitudinal wave numbers $k_{z n}$.

When the waveguide is inhomogeneous along its length, e.g., by variations of the inner radius of the liner, $k_{z n}$ becomes a function varying with $z$. A controlled variation of the cross section with distance, e.g., by applying a taper, can enhance the gain of the CFEL [10]. Here, we consider random variations with distance $z$ of the inner radius of the liner. Since the outer radius is constant, this is equivalent to variations in the thickness of the liner. The effect of a random fluctuation in the inner radius $r_{d}(z)$ of the liner is twofold. First, the fluctuating radius varies the phase velocity of the wave. Second, it modifies the transverse mode profile. However, it was shown that, if the liner fluctuations are small and slowly varying with distance $z$, they mainly affect the phase velocity of the wave while the transverse mode profile remains approximately unchanged [8]. Thus, for small liner fluctuations, Eq. (1) can be written as

$$
\vec{E}(\vec{r}, t)=\sum_{n=1}^{\infty} \overrightarrow{\mathcal{E}}_{n}(\vec{r}) \hat{a}_{n}(z) e^{i \phi_{n}(z)} e^{i\left(k_{z n}(z) z-\omega t\right)} .
$$

Here the amplitude $\hat{a}_{n}(z)$ contains a contribution from the fluctuations as well as from the interaction with the electron beam. Solutions for $a_{n}(z)$ and $\phi_{n}(z)$ are well known for the ideal case in the absence of liner fluctuations and are obtained by solving the set of coupled Maxwell and Newton-Lorentz equations [7]. However, here, when liner fluctuations are present, one expects that the dynamics of the CFEL becomes modified and results in a smaller growth rate of the field $\vec{E}$ with $z$. More specifically, we obtain for the normalized field amplitude, defined as $\hat{a}_{n}^{\prime}(z)=\frac{e}{m c_{0}} \hat{a}_{n}(z) e^{i \phi_{n}(z)}$, the following expression:

$$
\begin{aligned}
\sqrt{k_{z n}(z)}\left(1-\frac{\kappa_{n}^{2}}{k_{0 n}^{2}}\right) \frac{d \hat{a}_{n}^{\prime}}{d z}= & -2 \frac{\omega_{p}^{2}}{c_{0}^{2}} \frac{\beta_{z 0}}{A_{n} r_{d 0}^{2}} \\
& \times \int_{0}^{r_{d 0}} d r r\left[i I_{1}\left(\kappa_{n} r\right)\left\langle\frac{\beta_{r}}{\left|\beta_{z}\right|} e^{i \alpha_{n}}\right\rangle\right. \\
& +\frac{\kappa_{n}}{k_{0 n}} I_{0}\left(\kappa_{n} r\right)\left\langle e^{\left.\left.-i \alpha_{n}\right\rangle\right]}\right.
\end{aligned}
$$

Here $A_{n}$ is a normalization constant, $\omega_{p}$ is the plasma frequency, $\beta_{z 0}$ is the average initial electron velocity nor- malized to the speed of light in vacuum, $I_{0}$ and $I_{1}$ are the Bessel functions of second kind, and $\kappa_{n}$ is the transverse wave number. The symbol $\langle\cdots\rangle$ represents an average over all electrons within one radiation wavelength. Further symbols and the equations for the motion of the electrons are described in [8]. The variation of the phase velocity can be expressed as a variation of the longitudinal wave number $k_{z n}$ with $z$. To better display the scaling of the wave number, we expand the variation of $k_{z n}$ into a product of partial derivatives:

$$
\frac{d k_{z n}}{d z}=\frac{\partial k_{z n}}{\partial r_{d}} \frac{\partial r_{d}}{\partial z}
$$

The first factor $\left(\partial k_{z n} / \partial r_{d}\right)$ is a frequency dependent scaling factor. For a given frequency, it depends on the thickness and on the dielectric constant of the liner, and is thus specific for a particular design of the CFEL. The second factor $\left(\partial r_{d} / \partial z\right)$ contains the variations of the radius of the liner. Equation (4) makes clear that the size of phase velocity fluctuations, and thus the sensitivity of a CFEL to liner fluctuations, can be rather different for each CFEL even when the same absolute size of liner radius fluctuations is present. Therefore, to avoid a large sensitivity of the laser to liner fluctuations, geometries should be chosen that minimize the scaling factor $\partial k_{z n} / \partial r_{d}$. In general, the absolute value of the scaling factor, $\left|\partial k_{z n} / \partial r_{d}\right|$, is small for both very thin liners and for very thick liners. Very thin liners usually result in a high phase velocity (close to $c_{0}$ ), and thus require high energy electron beams. To minimize phase velocity fluctuations in low energy CFELs, the liner thickness should be as large as possible, while still maintaining the desired output and tuning characteristics.

Numerical integration of (3) shows that, with a sufficiently strong electron current (scaling with $\omega_{p}$ ), a weak input field (at $z=0$ ) of power $P_{\text {in }}$ undergoes an exponential growth until the growth saturates and terminates at a power $P_{\text {sat }}$ at a certain interaction length, $z_{\text {sat }}$. For a compact CFEL with high output, it is desirable to reach a high saturated power within a short distance $z_{\mathrm{sat}}$. From here on, we will thus use the parameter $P_{\text {sat }}$ and $z_{\text {sat }}$ to compare CFELs which are pumped with various currents, and in which different strengths of liner fluctuations are present.

\section{LOW- AND HIGH-GAIN OPERATION}

It is expected that different gain regimes have different sensitivities towards the effects of liner radius fluctuations. To study the influence of liner fluctuations on the output characteristics of CFELs in various regimes, we used for our calculations the parameters of a CFEL from a previous experiment [11], which can be operated with both low and high pump currents. The dependency on the gain of the device can then be studied by lowering the pump density, i.e., by lowering the current density in the device. As a typical example for the generation of an output frequency in the range of tens of $\mathrm{GHz}$, we assume the following 
parameters: the radius of the cylindrical electron beam is fixed at $r_{b}=5 \mathrm{~mm}$ and the electron-beam current $I_{b}$ is varied from 0.8 to $30 \mathrm{~A}$, corresponding to electron current densities of $1 \mathrm{~A} / \mathrm{cm}^{2}$ to $38 \mathrm{~A} / \mathrm{cm}^{2}$. The upper limit of $30 \mathrm{~A}$ is chosen to remain within the validity of our model, which neglects space-charge effects. We assume that the liner is made of alumina, which has a relative dielectric constant of $\epsilon_{r}=9.8$, with an average inner radius of $r_{d 0}=5.5 \mathrm{~mm}$ and with a constant outer radius of $r_{g}=6.07 \mathrm{~mm}$.

With the parameters given above and with no liner fluctuations, we have calculated the output power of the CFEL as a function of the seed frequency for a fixed accelerating voltage of $V_{b}=84.2 \mathrm{kV}$ and three values of the electron-beam current, $I_{b}=0.8 \mathrm{~A}, I_{b}=5 \mathrm{~A}$, and $I_{b}=$ $20 \mathrm{~A}$. For frequencies below $31.3 \mathrm{GHz}$, the phase velocity of the wave in the cold waveguide is above $c_{0}$ and, therefore we chose $32 \mathrm{GHz}$ as the lower frequency limit of the input seed in our calculations. The result is shown in Fig. 1. For the three examples shown here, it can be seen that there is a frequency for which the saturated power is maximum, which we term here $f_{\max }$. Figure 1 shows that for the accelerating voltage of $84.2 \mathrm{kV}, f_{\max }$ is just above $50 \mathrm{GHz}$. This frequency can be tuned by varying the accelerating voltage of the electron beam. The tuning range is displayed in Fig. 2(a) for beam voltages ranging from 60 to $100 \mathrm{kV}$ and a beam current of $0.8 \mathrm{~A}$. This figure shows $f_{\max }$ and the FWHM of the corresponding gain curve as a function of the beam voltage. It can be seen that $f_{\max }$ decreases with increasing accelerating voltage. In contrast, the FWHM is almost constant and only slightly increases for the lower accelerating voltages used in the calculations. Since we are interested both in the low- and the high-gain regimes of the CFEL, the frequency of maximum saturated power and the FWHM are calculated

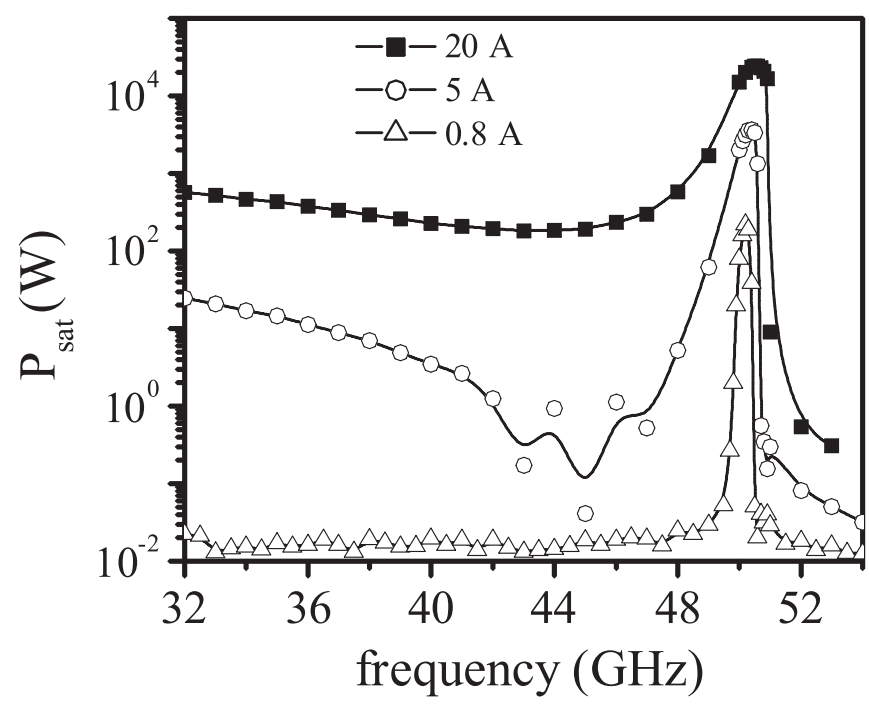

FIG. 1. Saturated power versus the frequency of the seed field for the electron-beam currents of $0.8,5$, and $20 \mathrm{~A}$ and no fluctuations in the liner radius. Lines are for guidance.
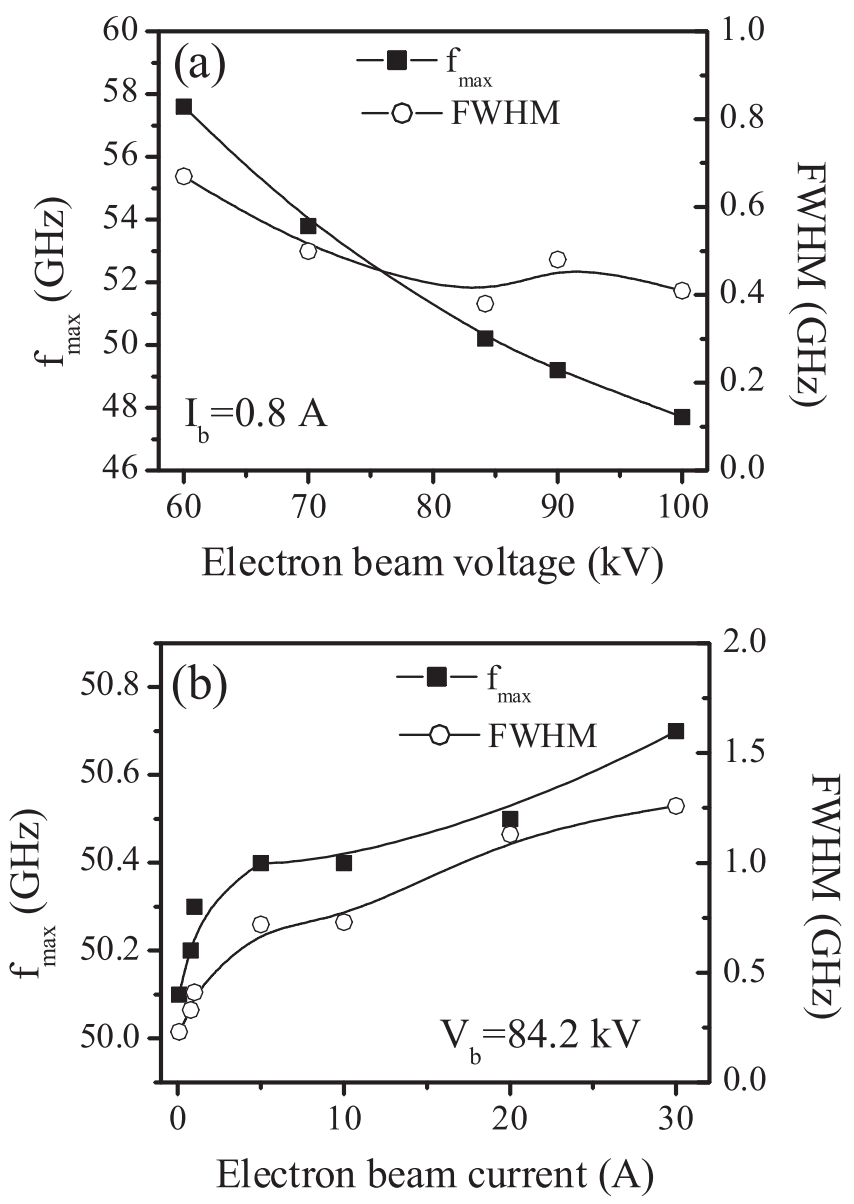

FIG. 2. Frequency of maximum saturated power and FWHM versus electron-beam accelerating voltage for $I_{b}=0.8 \mathrm{~A}$ (a), and versus electron-beam current for $V_{b}=84.2 \mathrm{kV}$ (b). Lines are for guidance.

for different electron-beam currents ranging from 0.8 to $30 \mathrm{~A}$ and a beam voltage of $V_{b}=84.2 \mathrm{kV}$. The result is shown in Fig. 2(b). It can be seen that both $f_{\max }$ and the FWHM increase for increasing electron-beam current. Although the FWHM increases by a factor of about 5 when the beam current is increased from 0.8 to $30 \mathrm{~A}$, it only gives an impression of the gain behavior near resonance. However, Fig. 1 shows that at strong pumping $(20 \mathrm{~A})$ there is a wide tail of appreciable power (of about a few hundreds of watts) from $32 \mathrm{GHz}$ to about $48 \mathrm{GHz}$, which are frequencies far below resonance. Also for the intermediate beam current of $5 \mathrm{~A}$, an appreciable output power (a few watts) can be observed from $32 \mathrm{GHz}$ to about $48 \mathrm{GHz}$. To better understand the latter effect, we have calculated the phase velocity of the amplified wave as a function of the probe frequency, for two cases corresponding to weak $(0.8 \mathrm{~A})$ and strong pumping $(20 \mathrm{~A})$, respectively. The results are plotted in Fig. 3(a). For comparison, we have also plotted the phase velocity in the cold waveguide as the dotted line and the velocity of the electrons as the solid line (velocities are normalized to $c_{0}$ ). It can be 


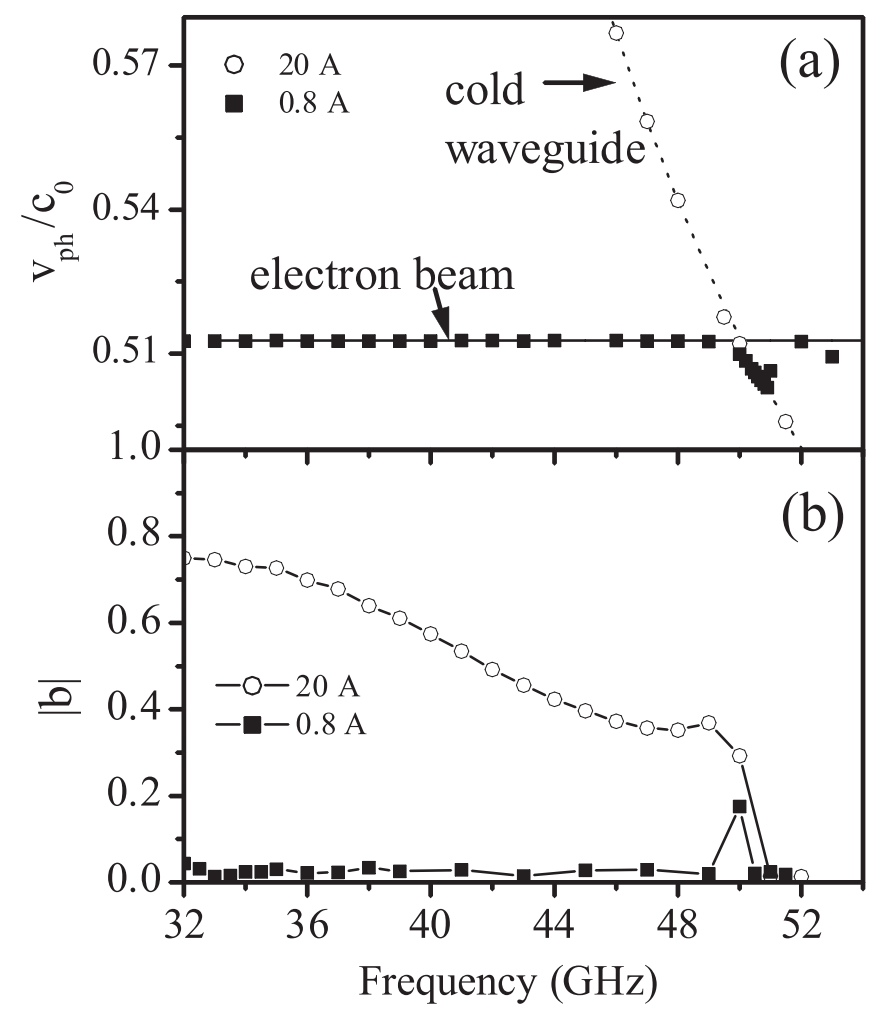

FIG. 3. Phase velocities (a) and modulus of the bunching (b) versus the frequency of the seed field for the electron-beam currents of 0.8 and $20 \mathrm{~A}$, and with no fluctuations in the liner radius. Part (a) also shows the initial velocity of the electron beam and the phase velocity of the $\mathrm{TM}_{01}$ mode in the cold waveguide. Lines are for guidance.

seen that at low pump current $\left(I_{b}=0.8 \mathrm{~A}\right)$, the phase velocity of the field is almost equal to the phase velocity of the cold $\mathrm{TM}_{0 n}$ mode and thus matches the electron velocity only in a narrow range above $50 \mathrm{GHz}$, resulting in a narrowband gain. In contrast, for strong pumping $\left(I_{b}=20 \mathrm{~A}\right)$, the phase velocity of the field is strongly reduced to a constant value matching the electron velocity over a wide range of frequencies spanning at least from $32 \mathrm{GHz}$ to above $50 \mathrm{GHz}$. Additionally, in a small range around $50 \mathrm{GHz}$, the phase velocity follows the velocity in the cold waveguide.

To explain the origin of the gain at frequencies below resonance, we use Eq. (3) that describes the dynamics of the wave in the CFEL. Equation (3) can be simplified by assuming $\beta_{r} \ll \beta_{z}$, i.e., the transversal velocity of the electrons is much smaller than the longitudinal velocity, so that we can neglect the first term in the right-hand side. Furthermore we assume, for simplicity, that the bunching is the same at every position within the cross section of the beam. Considering the case of no liner fluctuations this brings Eq. (3) into the following form:

$$
\frac{d \tilde{a}_{n}(z)}{d z}=-F\left\langle e^{-i \alpha_{n}(z)}\right\rangle,
$$

where $F$ is proportional to the form factor, which describes the overlap between the wave and electron beam. Note that $F$ is proportional to the plasma frequency $\omega_{p}^{2}$ and, therefore, $F$ is proportional to the current density within the electron beam. By splitting Eq. (5) into real and imaginary parts, we obtain the evolution of the CFEL amplitude and phase as

$$
\begin{gathered}
\frac{d a_{n}(z)}{d z}=-F\left\langle\cos \left[\alpha_{n}(z)+\phi_{n}(z)\right]\right\rangle \\
\frac{d \phi_{n}(z)}{d z}=\frac{F}{a_{n}(z)}\left\langle\sin \left[\alpha_{n}(z)+\phi_{n}(z)\right]\right\rangle .
\end{gathered}
$$

Equations (6) and (7) show that the amplification of the wave is driven by the complex bunching term $b=$ $\left\langle e^{i\left[\alpha_{n}(z)+\phi_{n}(z)\right]}\right\rangle$. When $b$ is real, only the amplitude is driven and the phase velocity of the wave remains equal to the phase velocity of the cold waveguide. On the other hand, when the bunching $b$ is imaginary, the bunching only drives the phase of the wave and, therefore, the phase velocity of the wave, which is given by

$$
v_{\phi_{n}}=\frac{\omega}{k_{z n}+\frac{d \phi_{n}(z)}{d z}}
$$

deviates from the value for a cold waveguide. In intermediate cases, where $b$ has both a real and imaginary part, the bunching term $b$ drives both the amplitude and the phase of the wave.

The maximum growth rate is thus found at a frequency $f_{\max }$ for which $d \phi_{n}(z) / d z=0$, i.e., when the phase velocity $v_{\phi_{n}}$ equals the phase velocity of the wave in the cold waveguide. In this case, $b$ is real. This is true for both lowand high-gain systems, i.e., systems pumped by small and large current densities. Consider now a frequency below $f_{\max }$. Figure 3(a) shows that the phase velocity of the cold waveguide is significantly higher than the electron-beam velocity. For low electron-beam current densities, the scaling factor $F$ in Eqs. (6) and (7) is small and therefore a growing amplitude $a_{n}(z)$ quickly stops the phase change. As a result, the phase velocity remains equal to the phase velocity of the wave in the cold waveguide, as we can see in Fig. 3(a) for the case $I_{b}=0.8 \mathrm{~A}$. Therefore, it is not possible to phase match the electrons to the wave and the gain quickly drops to zero for frequencies away from $f_{\max }$. The situation is different at high-current densities, where $d \phi_{n}(z) / d z$ can differ significantly from zero at high field amplitudes. In this case, the wave phase velocity is almost matched to the velocity of the electron beam over a wide range of frequencies, from $32 \mathrm{GHz}$ to about $48 \mathrm{GHz}$, as can be seen in Fig. 3(a). The result is an appreciable gain for this range of frequencies for high-current pumping as shown in Fig. 1 for $I_{b}=20 \mathrm{~A}$. Via Eqs. (6) and (7), the magnitude of the bunching determines the strength of both the wave amplification (or damping) and of the driving of the field phase. The modulus of the bunching term as a 
function of the frequency is calculated for 0.8 and $20 \mathrm{~A}$ and it is shown in Fig. 3(b). It can be seen that, for a high current, the bunching modulus increases for decreasing frequencies, probably due to a better overlap between the wave and the electron beam. For decreasing frequency, the difference in velocities between the wave in the cold waveguide and the electrons is larger as can be seen in Fig. 3(a). The slight increase in $b$ and the relative phase of $b$ with respect to the wave results in a sufficiently large $d \phi / d z$ to overcome this difference. Apparently, the increase in $b$ is sufficiently large that the growth rate increases as well resulting in a higher amplification for decreasing frequency.

The gain curve of Fig. 1 shows no significant gain at frequencies above $f_{\max }$. To understand this asymmetry we consider a frequency above $f_{\max }$. Here, the phase velocity of the wave in the cold waveguide is below the electronbeam velocity. Again, the electron beam drives the phase of the wave increasing the wave velocity to match the electron-beam velocity [see Fig. 3(a) for $I_{b}=20 \mathrm{~A}$ ]. However, the bunch is now formed at a phase that corresponds to attenuation of the radiation field and, therefore, no substantial gain is found at these frequencies.

\section{INFLUENCE OF LINER FLUCTUATIONS ON THE GAIN}

To model the variation in the inner liner radius, we use a random fluctuation of the radius along $z$, superimposed on the average radius $r_{d 0}$, and where in each specific distribution the strength of the liner fluctuations is characterized by its rms value, $\sigma_{r d}$. A spatial low-pass filter with a cutoff length $z_{c}$ is used to remove spatially fast fluctuations, compared to the wavelength, from the random distribution, which are excluded by the approximations used in our model (see [8]). We choose a cutoff length of $z_{c}=$ $10 \mathrm{~cm}$, such that this approximation is well fulfilled. The size of the rms value was set to values in a range between zero and $50 \mu \mathrm{m}$, which for this setup corresponds to a maximum tolerance in the radius of $1 \%$. Although this maximum tolerance seems small, it corresponds to an rms variation of almost $10 \%$ in the liner thickness. Therefore, this maximum is expected to induce large phase velocity fluctuations. Indeed, for the specific CFEL studied here we have that $\partial k_{z n} / \partial r_{d}=-4.6 \times 10^{6} \mathrm{~m}^{-2}$, and using Eq. (4), it can be calculated that the $50 \mu \mathrm{m}$ difference in $r_{d}$ produces a change in the longitudinal wave number of $\Delta k_{z n}=253 \mathrm{~m}^{-1}$. Therefore, a change of the liner radius of $1 \%$ results in a phase difference of $\pi$ when the wave propagates over a distance of only $1.3 \mathrm{~cm}$. Such a fluctuation in the liner radius is thus sufficient to move the electron bunch out of the gain region in this laser.

For the system described in the previous section, we calculate the saturated power $P_{\text {sat }}$ and the distance to saturation $z_{\text {sat }}$ when the liner radius fluctuations are present. In order to obtain statistical information of the output as a function of liner fluctuations, we generate an ensemble of 100 different realizations of random liner fluctuations for each combination of the rms fluctuations amplitude $\sigma_{r d}$ and cutoff distance $z_{c}$. For each combination we determine the ensemble average $\overline{P_{\text {sat }}}$ and its standard variation, $\sigma_{P}$, and the corresponding values for the distance to saturation, $\overline{z_{\mathrm{sat}}}$ and $\sigma_{z}$. A large standard deviation, $\sigma_{P}$ or $\sigma_{z}$, would indicate that in such a CFEL liner fluctuations should be avoided because the gain and the optimum interaction length are difficult to predict.

To study the effect of the liner fluctuations on the whole gain bandwidth of the laser, we calculate the saturated power and the distance to reach saturation for resonance and for frequencies below and above resonance. For resonance, Figs. 4(a) and 4(b) show the ensemble average of the saturated power, $\overline{P_{\text {sat }}}$, normalized to the saturated power in absence of fluctuations, $P_{0}$, as a function of the standard deviation of the liner fluctuations $\sigma_{r d}$. Figures 4(a) and 4(b) show the data for the electronbeam currents of 0.8 and $20 \mathrm{~A}$, respectively. The corresponding values for the distance to saturation $z_{\text {sat }}$ are shown in Fig. 5. In Fig. 4, it can be seen that the power for the low-gain device is reduced to almost zero already for small liner fluctuations, $\sigma_{r_{d}}=20 \mu \mathrm{m}$, which is only about $0.4 \%$ of the liner radius. Therefore, seemingly irrele-

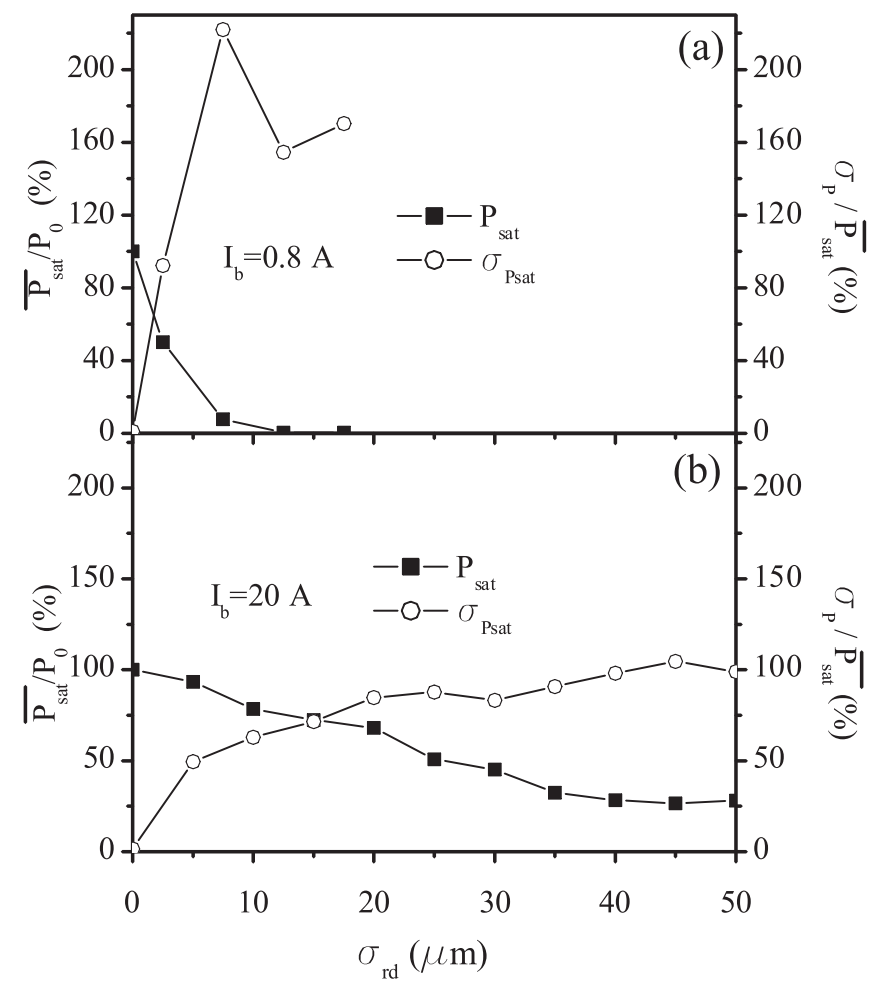

FIG. 4. Normalized saturated power $\left(\overline{P_{\text {sat }}} / P_{0}\right)$ and normalized standard deviation $\left(\sigma_{P} / \overline{P_{\text {sat }}}\right)$ as a function of the standard deviation of the liner fluctuations $\sigma_{r d}$ for a weak pump current of $0.8 \mathrm{~A}$ (a) and a strong pump current of $20 \mathrm{~A}$ (b), at a frequency of $50.2 \mathrm{GHz}$. The lines are for guidance. 


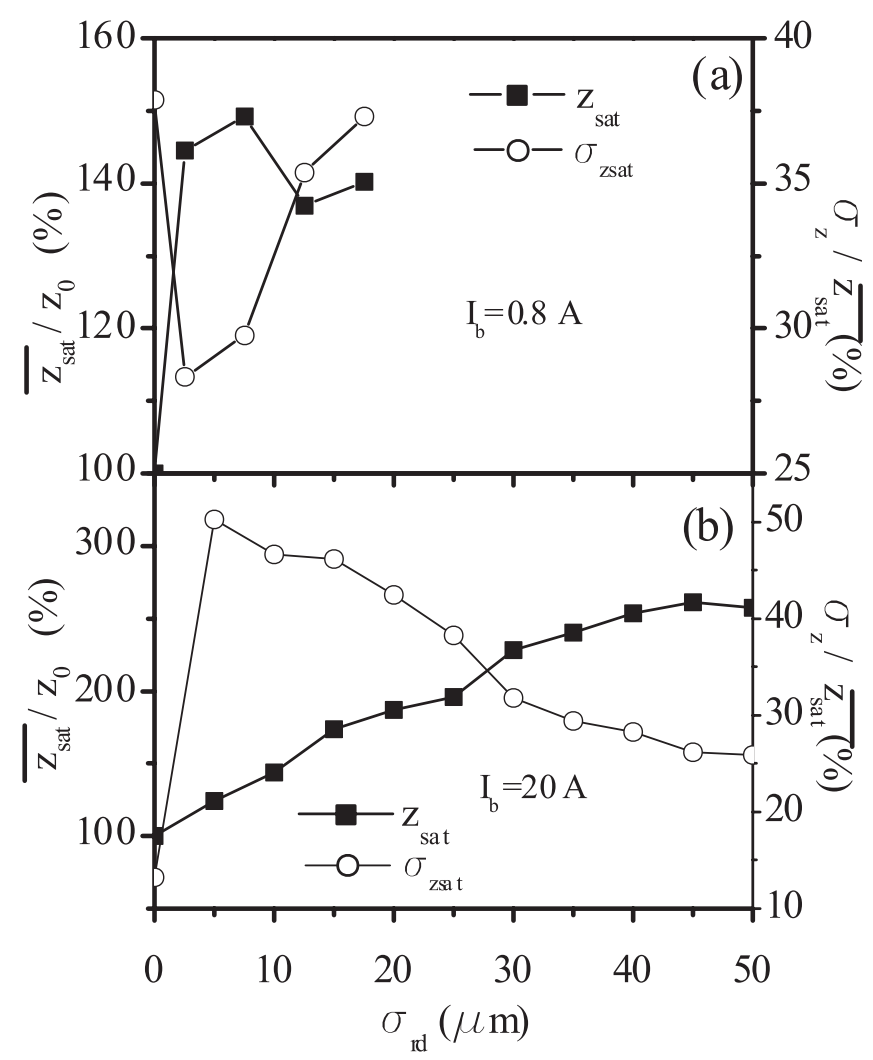

FIG. 5. Normalized distance to saturation $\left(\overline{z_{\mathrm{sat}}} / z_{0}\right)$ and normalized standard deviation of $z_{\mathrm{sat}}\left(\sigma_{z} / \overline{z_{\mathrm{sat}}}\right)$ as a function of the standard deviation of the liner fluctuations $\sigma_{r d}$ for a weak pump current of $0.8 \mathrm{~A} \mathrm{(a)}$ and a strong pump current of $20 \mathrm{~A} \mathrm{(b)}$, at a frequency of $50.2 \mathrm{GHz}$. The lines are for guidance.

vant liner fluctuations may lead to a complete failure in bringing this CFEL into operation. Note, however, that $\sigma_{r_{d}}=20 \mu \mathrm{m}$ is about $3.5 \%$ of the liner thickness. In contrast, with the same rms fluctuation but with strong pumping, almost $70 \%$ of the output (compared to the case of a perfect liner) can be expected. In both regimes of operation of the CFEL (weak and strong pumping), $\overline{z_{\mathrm{sat}}}$ is larger than $z_{0}$. Such increase of the distance to reach saturation is due to both the increase of the lethargy distance and the reduction of the growth rate of the CFEL. Thus, both the formation of the bunches and the phase matching are adversely affected by the liner fluctuations.

The results for frequencies away from resonance are given in Fig. 6(a), which shows the averaged saturated power $\overline{P_{\text {sat }}}$ as a function of the frequency of the seed field (from 32 to $54 \mathrm{GHz}$ ) for a standard deviation of the fluctuations in the liner radius of $\sigma_{r d}=20 \mu \mathrm{m}$. By comparison with the spectrum shown in Fig. 1, it can be seen that the fluctuations broaden the gain bandwidth of the CFEL and at the same time reduce the maximum saturated power. This is in agreement with the results shown in Fig. 4(b). This broadening initially increases the output at neighboring frequencies before the saturated power is also

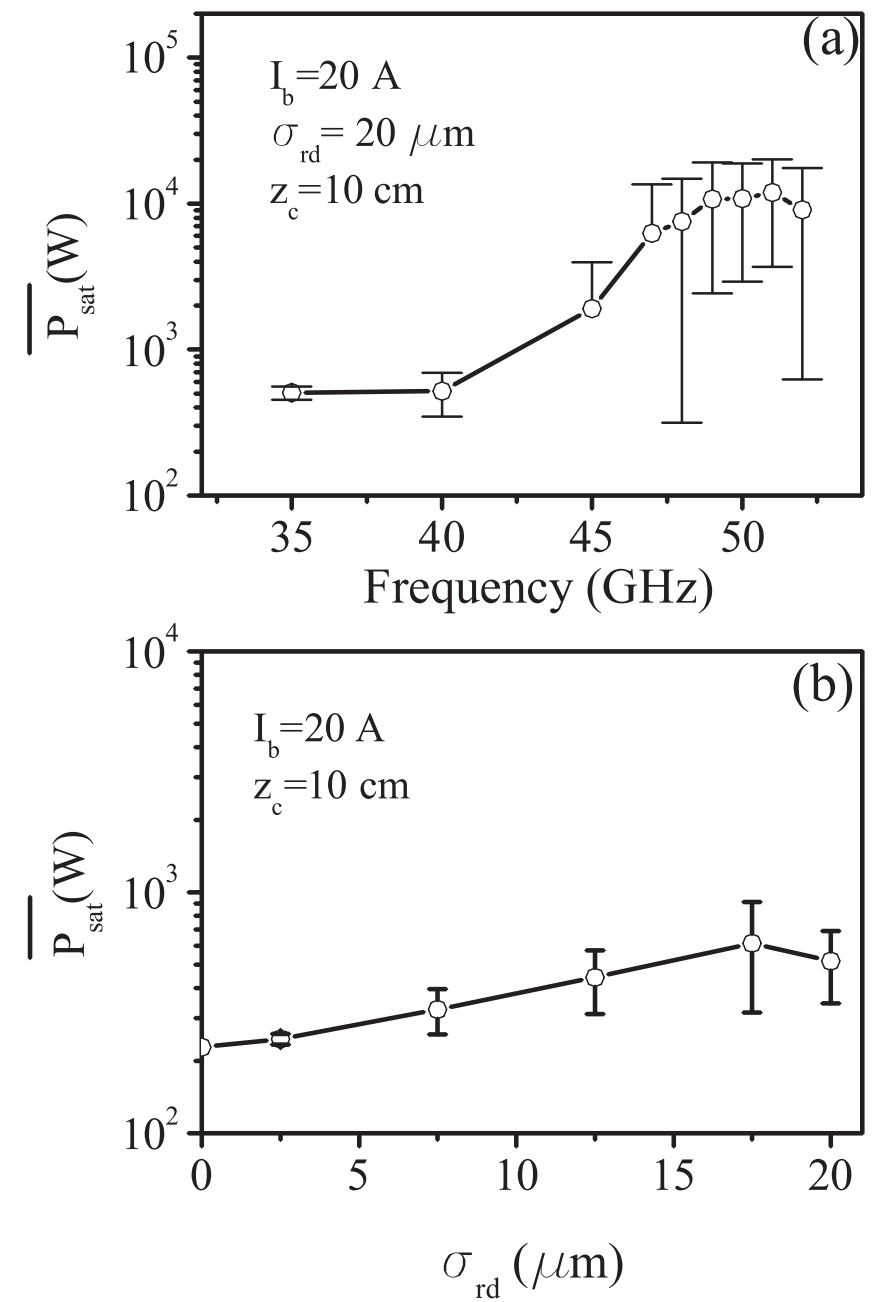

FIG. 6. For the electron-beam current of $20 \mathrm{~A}$, (a) average saturated power $\overline{P_{\text {sat }}}$ versus the frequency of the seed field for liner fluctuations characterized by $\sigma_{r d}=20 \mu \mathrm{m}$ and $z_{c}=$ $10 \mathrm{~cm}$ and (b) average saturated power $\overline{P_{\text {sat }}}$ versus $\sigma_{r d}$ for a frequency of $40 \mathrm{GHz}$. In both graphs lines are for guidance.

reduced at these frequencies. As an example, Fig. 6(b) shows the average saturated power for $40 \mathrm{GHz}$ as a function of the standard deviation of the fluctuations $\sigma_{r d}$. The power initially increases with the fluctuations as a result of the broadening. Eventually, for larger fluctuations, the power starts to drop here as well.

From the previous sets of results, we have seen that the reduction of the power for a fixed value of liner fluctuations is smaller the higher the current is. However, in an experimental setup, it is less convenient to use high currents. In order to know the minimum current necessary to avoid an excessive decrease of the power, we have calculated for a fixed value of liner fluctuations, $\sigma_{r_{d}}=15 \mu \mathrm{m}$, the saturated power as a function of the electron-beam pump current. We found that the electron-beam current must be larger than $30 \mathrm{~A}$ to keep the reduction in $\overline{P_{\text {sat }}}$ to less than $10 \%$. It means that this CFEL demands a large electronbeam current to overcome the effect of the fluctuations. 


\section{DISCUSSION AND CONCLUSION}

We found that strong pumping of a CFEL not only results in a high saturated power near the resonant frequency, but that the CFEL can also provide significant gain far away from resonance. The behavior of the high- and low-gain CFEL is explained in terms of the complex bunching term associated with the interaction of the electrons with the radiation wave. The saturated power is affected by the presence of liner inhomogeneities. For the CFEL investigated here we found that the saturated power is reduced by a factor of 2 for liner fluctuations of only $0.5 \%$ and $0.05 \%$ for strong and weak pumping, respectively. In an earlier study we also considered a CFEL operating at $50 \mathrm{GHz}$ [8] driven by a $0.8 \mathrm{~A}$ electron beam with a $1 \mathrm{~mm}$ radius $\left(25 \mathrm{~A} / \mathrm{cm}^{2}\right.$ current density) and for which $\partial k_{z n} / \partial r_{d}=-4.6 \times 10^{5} \mathrm{~m}^{-2}$. We found that an rms fluctuation in the inner radius of about $5 \%$ was required to reduce the saturated power by a factor of 2 . If we take into account the larger value of $\partial k_{z n} / \partial r_{d}$ (by a factor of 10) for the CFEL of this study then we observe that for the same liner fluctuations the phase shifts are a factor of 10 different between them. We can conclude that both devices, under equal pumping strengths, produce the same drop of saturated power for the same phase shift of the wave.

While at strong pumping regular precision tubes can be used without seriously degrading the gain of the device under study, it becomes increasingly more difficult to obtain or manufacture liners with the required precision when the device is operating in the low-gain regime. The design of low-gain CFELs therefore require optimization of the single pass gain that includes the sensitivity to liner irregularities.

[1] J. Walsh, High-Power Microwave Sources (Artech House, Boston, 1987).

[2] K. L. Felch, K. O. Busby, R. W. Layman, and J.E. Walsh, Free Electron Generators of Coherent Radiation, Physics of Quantum Electronics, edited by S.F. Jacobs, H.S . Pilloff, M. Sargent, M. O. Scully, and R. Spitzer (Addison-Wesley, Reading, MA, 1980), Vol. 7, p. 301.

[3] E. Garate, R. Cook, P. Heim, R. Layman, and J. Walsh, J. Appl. Phys. 58, 627 (1985).

[4] W. Main, R. Cherry, and E. Garate, Appl. Phys. Lett. 55, 1498 (1989).

[5] J.E. Walsh, C.H. Shaughnessy, R. Layman, G. Dattoli, G. P. Gallerano, and A. Renieri, Nucl. Instrum. Methods Phys. Res., Sect. A 272, 132 (1988).

[6] J. B. Murphy and J.E. Walsh, IEEE J. Quantum Electron. 18, 1259 (1982).

[7] H. P. Freund, Phys. Rev. Lett. 65, 2993 (1990).

[8] I. de la Fuente, P.J.M. van der Slot, and K. J. Boller, J. Appl. Phys. 100, 053108 (2006).

[9] R.E. Collin, Foundations for Microwave Engineering (McGraw-Hill, New York, 1966).

[10] B. W. Gore and V. B. Asgekar, Phys. Scr. 53, 62 (1996).

[11] J. Wieland, J. Couperus, P. J. M. van der Slot, and W. J. Witteman, Nucl. Instrum. Methods Phys. Res., Sect. A 429, 17 (1999). 